\title{
¿Why Does Arakawa and Schubert's Convective Quasi-Equilibrium Closure Not Work? Mathematical Analysis and Implications
}

\author{
JUN-ICHI YANO \\ CNRM, Météo-France, and CNRS, UMR 3589, Toulouse, France \\ ROBERT S. PLANT \\ Department of Meteorology, University of Reading, Reading, United Kingdom
}

(Manuscript received 14 June 2019, in final form 24 September 2019)

\begin{abstract}
Arakawa and Schubert proposed convective quasi equilibrium as a guiding principle for the closure of convection parameterization. However, empirical experiences from operational implementation efforts suggest that its strict application does not work well. The purpose of the present paper is to explain mathematically why this closure does not work in practice, and to suggest that problems stem from physically unrealistic assumptions. For this purpose, the closure hypothesis is examined in its original form, and without imposing a condition of a positiveness to the convective mass fluxes. The Jordan sounding with idealized large-scale forcing is used for diagnosis purposes. The question is addressed from several perspectives including the completeness of the entraining-plume spectrum, and a singular vector decomposition of the interaction kernel matrix. The main problems with the quasi-equilibrium closure are traced to (i) the relatively slow response of shallower convective modes to large-scale forcing and (ii) detrainment at convection top producing strong cooling and moistening. A strict application of the convective quasi-equilibrium principle leads to a singular response of shallow convection. An explicit coupling of convection with stratiform clouds would be crucial for preventing this unrealistic behavior, recognizing that the reevaporation of detrained cloudy air is a relatively slow process.
\end{abstract}

\section{Introduction}

Closure is a key issue in the convection parameterization problem (cf. Yano et al. 2013). Convective quasi equilibrium, as originally proposed by Arakawa and Schubert [1974; see Yano and Plant (2012a) as a review], remains an important guiding principle for the convective closure even today (e.g., Zhang 2002, 2003; Donner and Phillips 2003; Bechtold et al. 2014), in spite of various criticisms (e.g., Houze and Betts 1981; Mapes 1997).

The quasi-equilibrium closure may be formally stated for a spectral form of mass-flux convection parameterization as

$$
\mathbf{K M}+\mathbf{F}=0 .
$$

๑ Denotes content that is immediately available upon publication as open access.

Corresponding author: Jun-Ichi Yano, jiy.gfder@gmail.com
Here, $\mathbf{K}$ is an interaction matrix (kernel in Arakawa and Schubert 1974) that describes the feedback from the mass-flux vector (spectrum) $\mathbf{M}$ onto the large-scale tendency of an instability measure known as the cloud work function; $\mathbf{F}$ is the spectrum of large-scale forcing for the cloud work function, which is also defined as a vector. The vector components correspond to convective plume types that represent a spectrum of convective towers. The cloud work function corresponds to the rate at which available potential energy is converted into convective kinetic energy, as normalized by the mass flux at the convection base (cf. Yano et al. 2005a). Here, the equilibrium assumption states that the total tendency vanishes. Generalizations of the quasi-equilibrium ideas are discussed by Yano and Plant (2016).

Equation (1.1) states that the convective response (first term) is always in balance with the large-scale forcing (second term). This closure is, intuitively speaking, physically sound, because the convective process is much faster than the large-scale processes. However, in spite of a series of subsequent efforts, this original form 
of the closure has never become fully operational, but only in variant forms (e.g., Moorthi and Suarez 1992). This study will explain why the formulation given by Eq. (1.1) is structurally difficult to implement as a closure from a mathematical point of view.

The original implementation (Lord and Arakawa 1980; Lord 1982; Lord et al. 1982) devoted much attention to maintaining positiveness of the convective mass fluxes, because only convective updrafts were considered. Unfortunately, in our opinion and as we will discuss below, a rather elaborate iteration procedure introduced for this purpose may have obscured some more basic issues with a strict convective quasiequilibrium closure.

The present study focuses on the closure problem exactly as given by Eq. (1.1) without any further restrictions. This strategy may be partially justified by considering negative mass fluxes as detraining downdrafts (i.e., time-reversed updrafts). Importantly, regardless of whether this reinterpretation stands or not, this simplification enables us to elucidate more clearly and cleanly some basic problems with Arakawa and Schubert's (1974) original convective quasi-equilibrium closure.

For the same reason, the original assumption of a spectrum of purely entraining plumes is maintained in the present study, because we believe it is important to establish a baseline. In the literature, the problems with the oversimplified entraining-plume hypothesis have been extensively discussed, and various alternative formulations have been proposed, as reviewed in, for example, de Rooy et al. (2013) and Yano (2014a). Analysis with a more elaborate plume model would be considered a future work.

A simple formulation for the terms in Eq. (1.1) is provided in the next section, and some basic demonstrations of the problems are made in section 3. The identified problems are investigated in section 4 by examining the completeness of the entraining-plume spectrum as well as the mathematical structure of the interaction (kernel) matrix.

The present paper focuses on a rather narrow question of mathematical difficulties with the original closure formulation by Arakawa and Schubert (1974). Various physical issues associated with this closure hypothesis as well as with the mass-flux formulation itself are extensively discussed in the literature. Some of these may be found in a review of quasi equilibrium by Yano and Plant (2012a), and more general issues associated with the mass-flux parameterization are covered by Plant and Yano (2015). In concluding, in section 5, the paper also turns to the physical implications from the present findings, also referring to background issues.

\section{Formulation}

\section{a. Data}

A tropical climatology based on the Jordan sounding (Jordan 1958) is adopted for specifying vertical profiles of temperature and moisture. The vertical resolution used for the profile data is $50 \mathrm{hPa}$ from 1000 to $200 \mathrm{hPa}$, and with a surface value at $1015 \mathrm{hPa}$ being separately given. Data are also available at the 175-, 150-, 125-, 100-, 80-, 60-, 50-, 40-, and 30-hPa levels.

We introduce idealized large-scale advective forcings defined by

$$
\begin{aligned}
& F_{L}[T]=-\bar{w}\left(\frac{\bar{T}}{\bar{\theta}}\right) \frac{d \bar{\theta}}{d z}, \\
& F_{L}\left[q_{v}\right]=-\bar{w} \frac{d \bar{q}_{v}}{d z}
\end{aligned}
$$

for temperature and moisture, respectively. Here, $\bar{T}, \bar{\theta}$, and $\bar{q}_{v}$ are the vertical profiles for the temperature, the potential temperature, and the moisture as provided by the Jordan sounding. The large-scale vertical velocity $\bar{w}$ in Eq. (2.1) is prescribed by

$$
\bar{w}(z)=\left\{\begin{array}{ccc}
w_{0} \sin \pi\left[\frac{p(z)-p\left(z_{T}\right)}{p_{0}-p\left(z_{T}\right)}\right] & \text { for } & p_{0} \geq p(z) \geq p\left(z_{T}\right) \\
0 & \text { for } \quad p\left(z_{T}\right)>p(z)
\end{array}\right.
$$

as a function of the pressure, $p(z)$, with $w_{0}=10^{-2} \mathrm{~m} \mathrm{~s}^{-1}$, and $p_{0}=1015 \mathrm{hPa}$ the surface pressure. Three types of large-scale forcing are considered: deep $\left(z_{T}=15 \mathrm{~km}\right)$, shallow $\left(z_{T}=5 \mathrm{~km}\right)$, and very shallow $\left(z_{T}=1.5 \mathrm{~km}\right)$. The purpose of this idealization is to examine the convective response to large-scale forcing strictly confined to a certain vertical range. These forcing profiles are shown in Fig. 1a. Here, as a drastic simplification, potential contributions to the forcing from boundary layer processes are neglected, despite their possible importance. Consistent with that assumption, contributions from boundary layer processes to the interaction matrix $\mathbf{K}$ will also be neglected in the analysis below.

The large-scale forcing on the cloud work function $\mathbf{F}$ from Eq. (1.1) is obtained by vertically integrating a linear combination of two large-scale forcings, as explicitly given by Eq. (B33) in Arakawa and Schubert (1974). The integration is defined with a weighting that is a function of the fractional entrainment rate $\varepsilon$ (see next subsection) and the resulting integrated forcing is presented in Fig. 1b. We remark that the forcing has a relatively weak dependence on a microphysical parameter $c_{0}$, which is defined by Eq. (2.5) below in section 2c. 

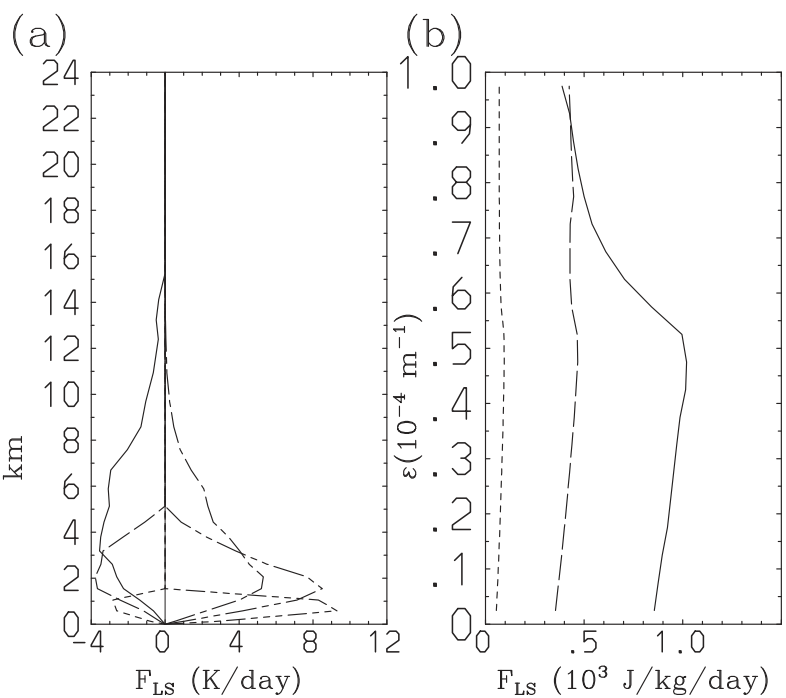

FIG. 1. The three types of the large-scale forcing profile considered: deep (solid and chain) dashed shallow (long dashed and double dotted-dashed), and very shallow (short dash and triple dotted-dashed). (a) The forcings are shown as a function of height for both the thermal (negative curves) and the moisture (positive curves) terms. (b) The forcings are shown in terms of the generation rate of cloud work function [as found in Eq. (1.1)] across the spectrum of fractional entrainment rates.

The vertical profile of the large-scale forcing as defined by Eqs. (2.1a), (2.1b), and (2.2) has a well-defined vertical scale but its projection onto the plume components in Fig. 1b, presents a very broad distribution of forcing as a function of the entrainment rate, despite the fact that the entrainment rate determines the vertical scale of each plume mode. Moreover, the main difference from changing the vertical scale of large-scale forcing is a change of the spectrum amplitude rather than a change of the spectrum shape.

We diagnose the convective quasi-equilibrium closure of Eq. (1.1) by closely following the mass-flux spectrum formulation introduced by Arakawa and Schubert (1974), and for formulation details we refer to the original paper. In the following two subsections, we describe two major assumptions for which some additional specifications are required: the entraining-plume spectrum (section $2 \mathrm{~b}$ ) and the precipitation formulation (section 2c).

\section{b. Entraining-plume spectrum}

Arakawa and Schubert's (1974) entraining-plume spectrum is characterized by a set of constant fractional entrainment rates $\varepsilon_{i}$, which are defined in this study by

$$
\varepsilon_{i}=\frac{i-1 / 2}{n} \varepsilon_{\max },
$$

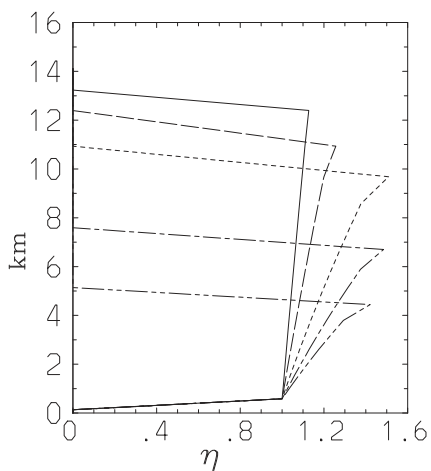

FIG. 2. Normalized mass-flux profiles, $\eta=M(z) / M\left(z_{B}\right)$, for selected entraining plumes under the microphysical formulation given by Eq. (2.5). In order from the deepest (solid) to the shallowest profiles (double-dotted chain), the plots are for values of $\varepsilon=1 \times 10^{-5}, 2 \times 10^{-5}, 4 \times 10^{-5}, 6 \times 10^{-5}$, and $8 \times 10^{-5} \mathrm{~m}^{-1}$.

where the vector index $i$ spans for $i=1, \ldots, n$ with $n=20$ plume types considered, and $\varepsilon_{\max }=10^{-4} \mathrm{~m}^{-1}$ is the maximum fractional entrainment rate considered. The $i$ th entraining plume has a normalized mass-flux profile of

$\eta_{i}(z)=\left\{\begin{array}{cl}\exp \left[-\varepsilon_{i}\left(z-z_{B}\right)\right] & \text { for } z_{B} \leq z \leq z_{T i}, \\ 0 & \text { otherwise }\end{array}\right.$

where $z_{B}$ and $z_{T i}$ are the bottom and top levels of the plume, respectively. The base level $z_{B}$ is taken to be $950 \mathrm{hPa}(583 \mathrm{~m})$, approximately corresponding to the top of the convectively well-mixed boundary layer. The top $z_{T i}$ is defined by the level of neutral buoyancy, at which all of the plume air detrains into the environment. The top height $z_{T i}$ is diagnosed as a continuous function by taking a linear interpolation of values between the data height levels and we assume that the plume-top detrainment happens over a vertical layer spanning between these two levels.

For a larger fractional entrainment rate $\varepsilon$, the inplume air is more diluted by the environmental air, and so becomes less buoyant. As a result, the plume top height $z_{T i}$ decreases with increasing $\varepsilon$. In essence, the fractional entrainment rate $\varepsilon$ becomes a reverse measure of the convection depth $z_{T}$. Some examples of vertical profiles of entraining plumes for the Jordan sounding are shown in Fig. 2. A full mass-flux profile for the $i$ th plume is defined by $M_{i} \eta_{i}(z)$, where $M_{i}$ is the mass flux at the plume base for the plume type and is the $i$ th component of the mass-flux vector $\mathbf{M}$ in Eq. (1.1).

\section{c. Precipitation efficiency}

A very simple cloud microphysics is used, in which the precipitation rate $R_{i}$ within the $i$ th plume at each vertical level is assumed to be proportional to the cloud water 


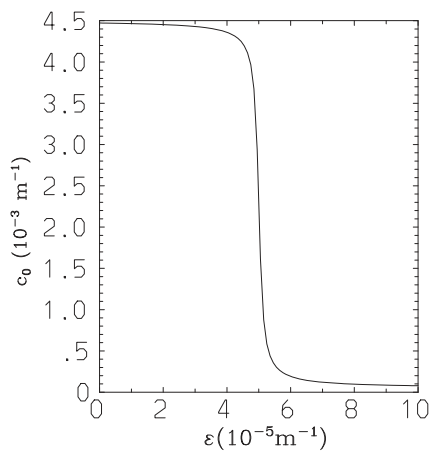

FIG. 3. Dependence of the precipitation efficiency $c_{0}$ on the fractional entrainment rate $\varepsilon$ as defined by Eq. (2.5).

vertical flux $w_{c i} q_{c i}$ with a proportionality constant $c_{0}$ called the precipitation efficiency:

$$
R_{i}=c_{0} w_{c i} q_{c i} .
$$

Here, $w_{c i}=M_{i} \eta_{i} / \rho \sigma_{i}$ and $q_{c i}$ are, respectively, the incloud vertical velocity and the cloud water mixing ratio of the $i$ th plume type, and $\rho$ is the air density. The precipitation rate $R_{i}$ is defined in such a manner that the fractional area $\sigma_{i}$ occupied by the $i$ th plume type does not appear in actual calculations of the total water for a given plume type [cf. Eq. (6.2b) of Yano 2015].

The precipitation efficiency $c_{0}$ is chosen by following a curve shown in Hack et al. (1984, their Fig. 3). Specifically, we take

$$
c_{0}=-\frac{2 \Delta c}{\pi} \arctan \left(\frac{\varepsilon-\varepsilon_{0}}{\varepsilon_{c}}\right)+c_{00},
$$

where $c_{00}=\left(c_{\max }+c_{\min }\right) / 2$ and $\Delta c=\left(c_{\max }-c_{\min }\right) / 2$. Note that $c_{0} \rightarrow c_{\max }$ and $c_{0} \rightarrow c_{\min }$ as $\varepsilon \rightarrow 0$ and $\varepsilon \rightarrow \infty$, respectively; $\varepsilon_{0}$ marks a transition from a weakly precipitating shallow (with large $\varepsilon$ ) regime to a heavily precipitating deep (with small $\varepsilon$ ) regime. Here, the parameters are set as $\varepsilon_{0}=5 \times 10^{-5} \mathrm{~m}^{-1}, c_{\max }=4.5 \times$ $10^{-3} \mathrm{~m}^{-1}, c_{\min }=5 \times 10^{-5} \mathrm{~m}^{-1}$, and $\varepsilon_{c}=10^{-6} \mathrm{~m}^{-1}$. Figure 3 plots the precipitation efficiency $c_{0}$ as a function of the fractional entrainment rate $\varepsilon$.

\section{Basic analyses}

\section{a. Interaction matrix}

The interaction matrix (kernel) $\mathbf{K}$ is defined by Eq. (B32) and Eqs. (B35)-(B38) of Arakawa and Schubert (1974). Its evaluation using Eq. (2.5) for the precipitation efficiency is shown in Fig. 4a. An element $K_{i j}$ of the interaction matrix defines the rate at which a unit of the convection-base mass flux for the $j$ th plume type $M_{j}$ changes the cloud work function for the $i$ th plume type.
By referring to Eq. (144) and Fig. 11 of Arakawa and Schubert (1974), we find that the large-scale thermodynamic profiles are modified by convection in two major ways: (i) detrainment at the plume top, which cools and moistens the large-scale environment due to evaporation of the detrained cloudy air, and (ii) a compensating descent in the large-scale environment, which leads to adiabatic heating and drying by downward transport of drier air from aloft. These two major processes modify the cloud work function, and the interaction matrix can be separated into two dominant contributions:

$$
\mathbf{K}=\mathbf{K}_{d}+\mathbf{K}_{v},
$$

where $\mathbf{K}_{d}$ and $\mathbf{K}_{v}$ represent the effects of detrainment and environmental descent, respectively. A third part $\mathbf{K}_{M}$ as defined by Eq. (B32) of Arakawa and Schubert (1974), is neglected because of our assumptions above about boundary layer processes.

The evaporative cooling associated with detrainment leads to a further destabilization of the atmosphere, and thus $\mathbf{K}_{d}$ is positive definite (Fig. $4 b$ ). This tendency is stronger when a plume is less strongly precipitating, and hence for the shallower plumes with larger $\varepsilon^{\prime}$. Moreover, the detrainment effect is felt only by the plume types that extend higher than the detrainment level of the plume in question (i.e., $\varepsilon<\varepsilon^{\prime}$ ), and so $\mathbf{K}_{d}$ is triangular. On the other hand, adiabatic heating by environmental descent leads to a stabilization, and thus $\mathbf{K}_{v}$ is negative definite (Fig. 4c). The descent effect is stronger for deeper plumes with smaller $\varepsilon^{\prime}$, and affects plume types of all depths.

\section{b. Response due to a single plume}

Once a value of $M_{i}$, as a component of the mass-flux vector $\mathbf{M}$, is specified [see also Eq. (3.2) below], the tendencies of temperature and moisture produced by each convective plume type, $i$, can be calculated, respectively, from Eqs. (3.6a) and (3.6b) of Yano (2015). Examples of the convective response from individual plume types are shown in Fig. 5. Here, we rather arbitrary assume $M_{i}=10^{-2} \mathrm{~kg} \mathrm{~m}^{-2} \mathrm{~s}^{-1}$. For the cases of $\varepsilon=6 \times 10^{-5}$ and $8 \times 10^{-5} \mathrm{~m}^{-1}$, the resulting plumes are relatively shallow, with relatively weak precipitation. This leads to strong cooling and moistening at the detrainment level associated with cloud evaporation. The effects are much less pronounced for the deep-plume example, because a high precipitation does not leave much cloud water for detrainment at the plume top. The values obtained for the strong cooling and moistening associated with the detrained-air reevaporation are shown in the appendix to be consistent with a simple scale analysis. 

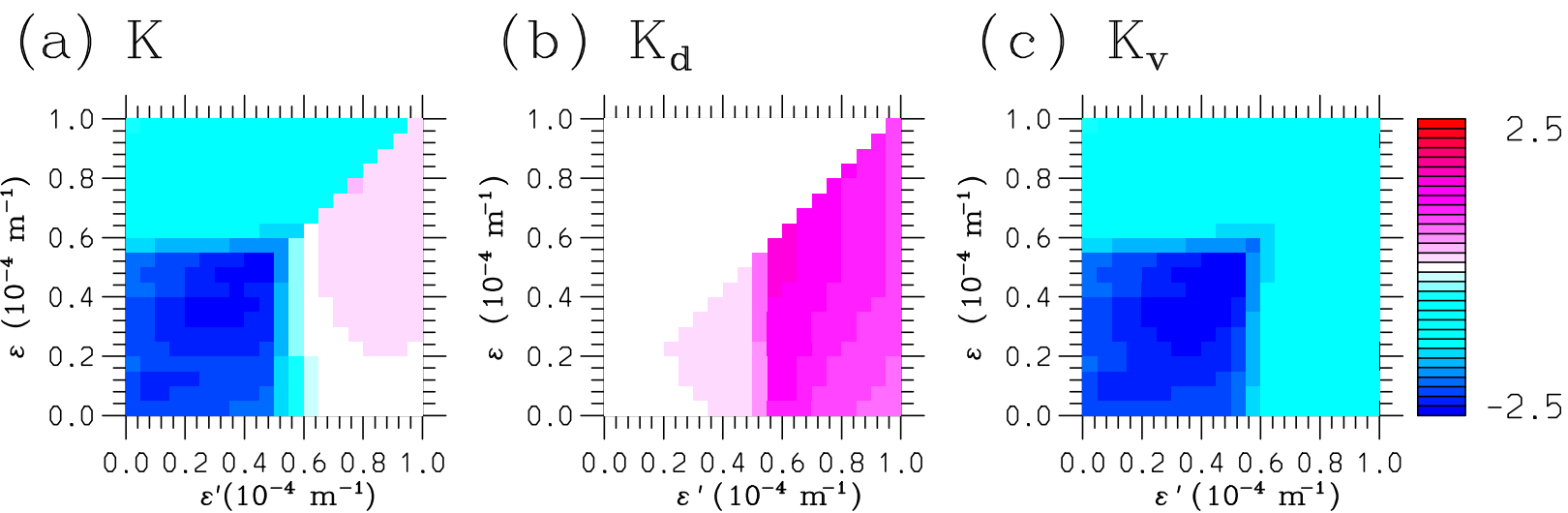

FIG. 4. The interaction matrix $\mathbf{K}_{i j}$ is plotted with the index $i$ shown vertically and $j$ shown horizontally for corresponding fractional entrainment rates $\varepsilon$ and $\varepsilon^{\prime}$, respectively, as defined by Eq. (2.3). (a) The full matrix and the two components due to (b) detrainment and (c) environmental descent are shown. The evaluation uses the deep large-scale forcing (solid and chain-dashed curves in Fig. 1).

The strongly peaked character of the thermodynamic tendencies from individual plume types raises potential issues for construction of the total convective response, obtained by taking a linear sum of these individual tendencies weighted by the convection-base mass-flux values, $M_{i}$. The total response is considered next.

\section{c. Total convective response}

The convective-base mass-flux vector, $\mathbf{M}=\left(M_{i}\right)$, is obtained from Eq. (1.1) by multiplying the inverted matrix $\mathbf{K}^{-1}$ on the large-scale forcing $\mathbf{F}$. The obtained $\mathbf{M}$, shown in Fig. 6a as a function of the fractional entrainment rate $\varepsilon$, is marked by relatively large contributions from both small and large $\varepsilon$ with modest contributions from intermediate values. This basic structure is not dependent on the depth of the large-scale forcing.

The resulting vertical profile of the total mass flux $M(z)$ is given by

$$
M(z)=\sum_{i=1}^{n} \eta_{i}(z) M_{i}
$$

and is shown in Fig. $6 \mathrm{~b}$, where $\eta_{i}(z)$ is the vertical profile of the $i$ th plume type, as defined by Eq. (2.4).

The most noticeable feature is a strong downdraft below the 4-km level, which is the lowest height achieved by plumes with largest fractional entrainment rates $\varepsilon$ under the given mean thermal profile. Above this level, a substantial updraft reaches the 14-km level under deep large-scale forcing (solid curve), consistent with the depth of forcing in Fig. 1a. It is replaced by an updraft that decreases linearly with height between 4 and $14 \mathrm{~km}$ under shallow large-scale forcing (long dashed). This response is rather unintuitive considering the fact that shallow large-scale forcing only reaches the $5-\mathrm{km}$ level (cf. Fig. 1). Only when very shallow large-scale forcing is considered does the convective response above the 4-km level becomes negligible (short dashed).

Figure 7 shows the corresponding convective tendency profiles for temperature (Fig. 7a) and moisture (Fig. 7b). Clearly these do not match well with the forcings in Fig. 1, even though the cloud work functions for each mode are in equilibrium by construction (cf. section 5a). The sudden increase of mass flux at the 4-km level (Fig. 6b) is associated with unrealistically strong heating and drying, with magnitudes circa $60 \mathrm{~K} \mathrm{day}^{-1}$ for temperature and $-120 \mathrm{~K} \mathrm{day}^{-1}$ for moisture. The peaks are manifestations of those seen for individual plume (a)

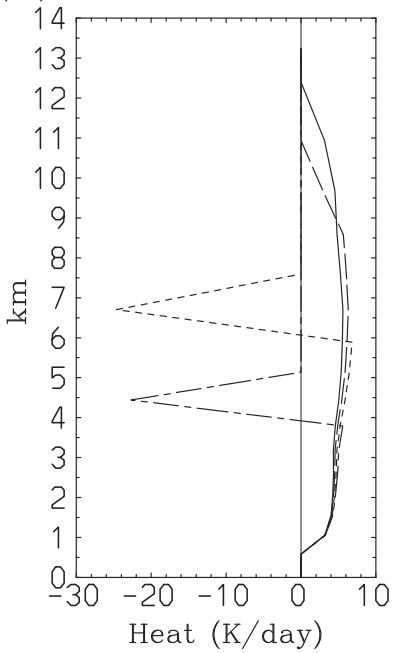

(b)

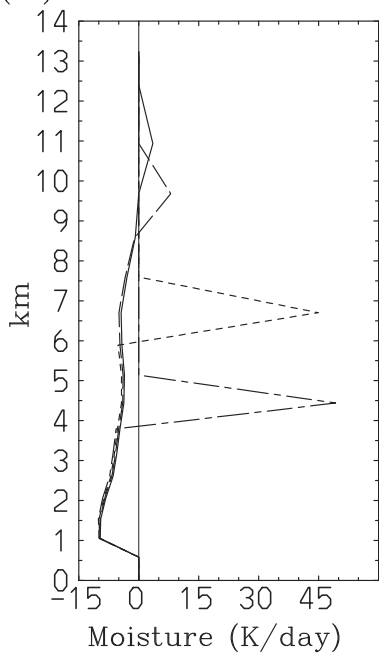

FIG. 5. Profiles of the tendencies of (a) the temperature and (b) the moisture (mixing ratio) produced by convective plumes for given, selected entrainment rates: $\varepsilon=2 \times 10^{-5}$ (solid), $4 \times 10^{-5}$ (long dashed), $6 \times 10^{-5}$ (short dashed), and $8 \times 10^{-5} \mathrm{~m}^{-1}$ (chain dashed). Plotted in units of $\mathrm{K} \mathrm{day}^{-1}$ and assuming the convective mass-flux amplitude of $M_{i}=10^{-2} \mathrm{~kg} \mathrm{~m}^{-2} \mathrm{~s}^{-1}$. 


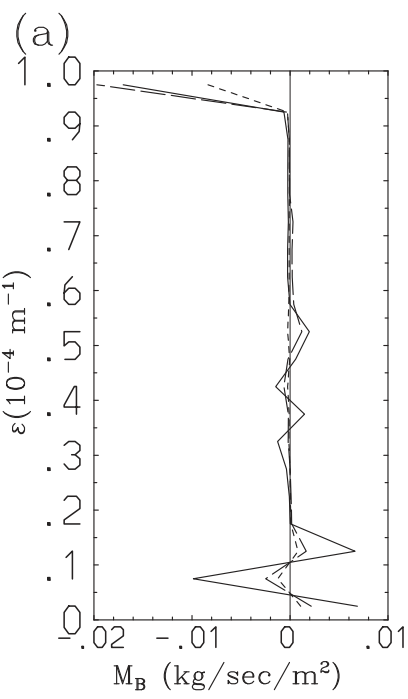

(b)

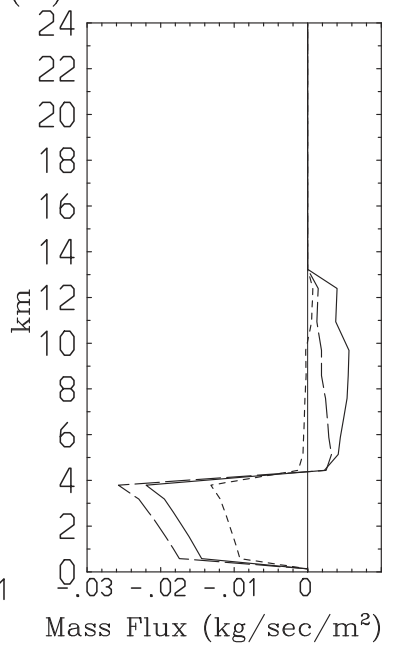

FIG. 6. (a) The spectrum of convective-base mass flux as a function of the fractional entrainment rate, as obtained from inverting the matrix $\mathbf{K}$ in Eq. (1.1). Results are presented for the deep (solid), shallow (long dashed), and very shallow (short dashed) forcings, as shown in Fig. 1. (b) The corresponding vertical profiles of the total mass flux.

types in Fig. 5, but with the signs reversed: entrainment (i.e., negative cloud-top detrainment) at the top of detraining-downdraft plumes causes this tendency. On the other hand, tendencies with more reasonable magnitudes are found at the other vertical levels.

\section{Further analyses}

\section{a. Completeness of the spectrum of plumes}

The basic idea of the spectrum model is to be able to represent every possible convective profile using a sum of profiles from the individual plumes. Thus, we now ask whether the ensemble of entraining plumes has such a capacity. The question may be more formally posed as the possibility of decomposing any given arbitrary massflux profile $M(z)$ by a plume spectrum given by the set of functions $\left\{\eta_{i}\right\}(i=1, \ldots, n)$ as

$$
M(z)=\sum_{i=1}^{n} \tilde{m}_{i} \eta_{i}(z)
$$

where $\tilde{m}_{i}$ are the expansion coefficients. Unfortunately, performing such a decomposition is not straightforward, because the exponential entraining-plume profiles of Eq. (2.4) do not constitute an orthogonal set. Nevertheless, it is instructive to consider the issues further by assessing decompositions of both $\eta_{i}$ and $M$ using a complete orthonormal set. For this purpose, it is (a)

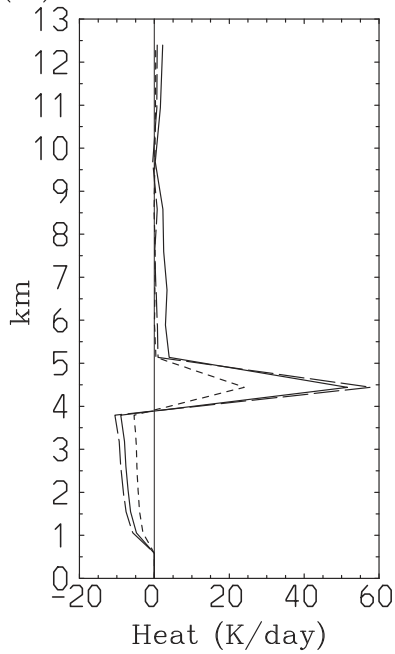

(b)

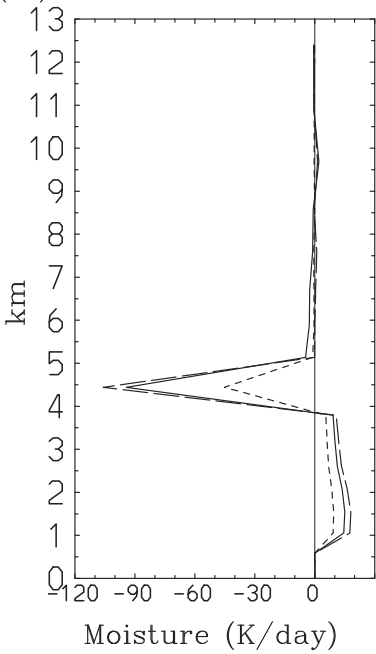

FIG. 7. Vertical profiles of the convective tendencies for (a) temperature and (b) moisture (the mixing ratio) for the three large-scale forcing profiles given in Fig. 1: deep (solid), shallow (long dashed), and very shallow (short dashed).

convenient to use the vertical normal modes $W_{i}(z)$ for the vertical velocity defined for the hydrostatic primitive equation system (Kasahara and Puri 1981; Fulton and Schubert 1985). Thus, we set

$$
\begin{aligned}
& \eta_{j}=\sum_{i=1}^{n} \hat{\eta}_{i j} W_{i}(z), \\
& M=\sum_{i=1}^{n} \hat{m}_{i} W_{i}(z)
\end{aligned}
$$

with expansion coefficients $\hat{\eta}_{i j}$ and $\hat{m}_{i}$ for $\eta_{j}$ and $M$, respectively. By substituting Eq. (4.2a) into Eq. (4.1), and by comparing this result with Eq. (4.2b), we find

$$
\hat{m}_{i}=\sum_{j=1}^{n} \tilde{m}_{j} \hat{\eta}_{i j}
$$

and so the expansion coefficients $\tilde{m}_{j}$ are determined by inverting the matrix $\hat{\eta}_{i j}$. In order for the inverse to exist, the determinant of this matrix must be nonzero.

To investigate the structure of the matrix, we perform a singular vector decomposition:

$$
\hat{\eta}_{i j}=\sum_{k=1}^{n} \lambda_{k} w_{i k} \tilde{w}_{k j}
$$

with eigenvalues $\lambda_{k}$ and eigenvectors $w_{i k}$ and $\tilde{w}_{k j}$, the subscript $k$ designating the index for the eigenmode. These are defined by linear eigenvalue problems: 
(a)

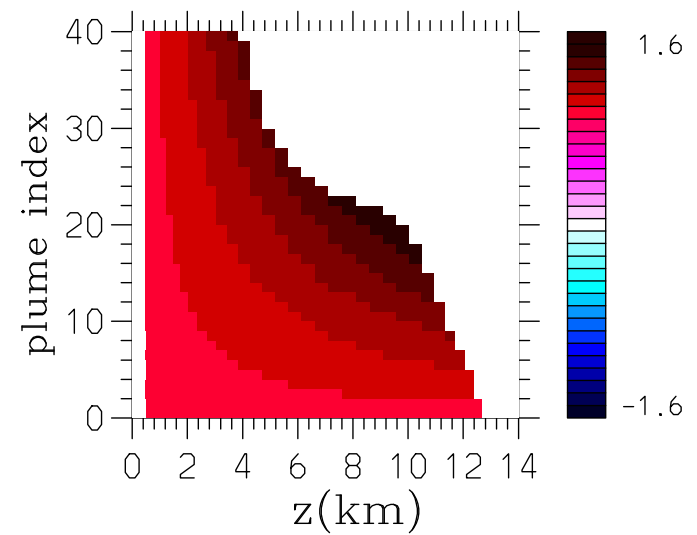

( b )

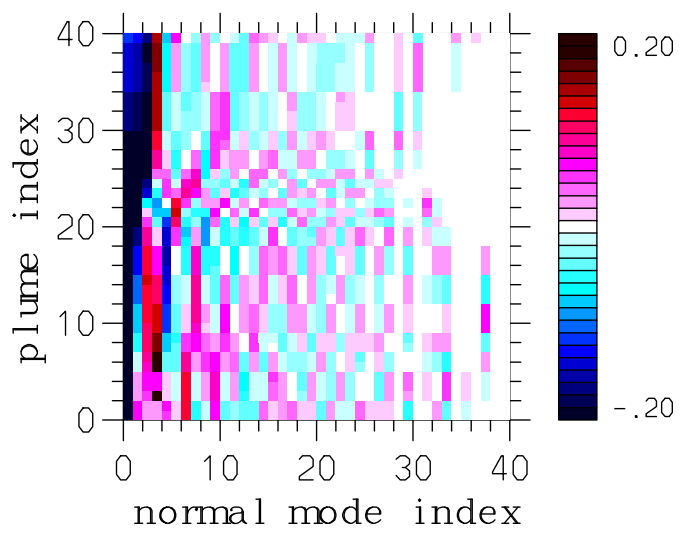

FIG. 8. (a) The vertical profiles for the plume spectrum $\left\{\eta_{j}(z)\right\}$, shown as a function of height (horizontal axis) and the plume index (vertical axis). (b) The plume matrix (i.e., the spectrum of plumes decomposed by the verticalvelocity normal modes) $\hat{\eta}_{i j}$, shown as a function of the normal mode index $i$ (horizontal axis) and the plume-type index $j$ (vertical axis). See Eq. (4.2a) for its definition.

$$
\begin{aligned}
& \sum_{j=1}^{n} \hat{\eta}_{i j} w_{j k}=\lambda_{k} w_{i k}, \\
& \sum_{i=1}^{n} \tilde{w}_{k i} \hat{\eta}_{i j}=\lambda_{k} \tilde{w}_{k j} .
\end{aligned}
$$

These two vector sets are called the right and the left vectors, which satisfy the orthonormality

$$
\sum_{k=1}^{n} \tilde{w}_{i k} w_{k j}=\delta_{i j}
$$

As a result, the determinant and the inverse matrix are defined by

$$
\begin{aligned}
\operatorname{det}\left(\hat{\eta}_{i j}\right) & =\prod_{k=1}^{n} \lambda_{k}, \\
\hat{\eta}_{i j}^{-1} & =\sum_{k=1}^{n} \lambda_{k}^{-1} w_{i k} \tilde{w}_{k j},
\end{aligned}
$$

respectively. The eigenvalue spectrum $\left\{\lambda_{k}\right\}$ characterizes a singularity of a given matrix. If any of the eigenvalues $\lambda_{k}$ are too small, the determinant becomes very small, and the inverse matrix becomes singular.

Figures $8 \mathrm{a}$ and $8 \mathrm{~b}$ shows the plume spectrum $\left\{\eta_{j}(z)\right\}$ and the plume matrix $\left(\hat{\eta}_{i j}\right)$, respectively. To ensure that we retain sufficient vertical modes for the decomposition, and henceforth for the dimension of the matrix $\left(\hat{\eta}_{i j}\right)$, we reset $n=40$ in Eq. (2.3) only for the analysis of the present subsection. The eigenvalues, $\lambda_{k}$, obtained by the singular vector decomposition of Eq. (4.4) are plotted in Fig. 9: note that we have chosen to label the eigenvectors in order of decreasing magnitude of the corresponding eigenvalues $\left|\lambda_{k}\right|$. The eigenvalues fall to very small values above $k \geq 20$, suggesting that the entraining-plume decomposition is highly redundant, and as a result the determinant of the matrix $\left(\hat{\eta}_{i j}\right)$ practically vanishes.

However, the singular vector decomposition can be used to regularize a matrix by removing all the small eigenvalues $\lambda_{k}$ with, say, $k>n_{c}$ (with $n_{c}<n$ ) from the summations in Eqs. (4.4) and (4.7b). Thus, we obtain

$$
\begin{gathered}
\hat{\eta}_{i j} \simeq \sum_{k=1}^{n_{c}} \lambda_{k} w_{i k} \tilde{w}_{k j}, \\
\hat{\eta}_{i j}^{-1} \simeq \sum_{k=1}^{n_{c}} \lambda_{k}^{-1} w_{i k} \tilde{w}_{k j} .
\end{gathered}
$$

Setting $n_{c}=16$ yields a regularized matrix $\left(\hat{\eta}_{i j}\right)$ shown in Fig. 10a, and its transformation back to real space leads to Fig. 10b. The reconstruction is noisier than the

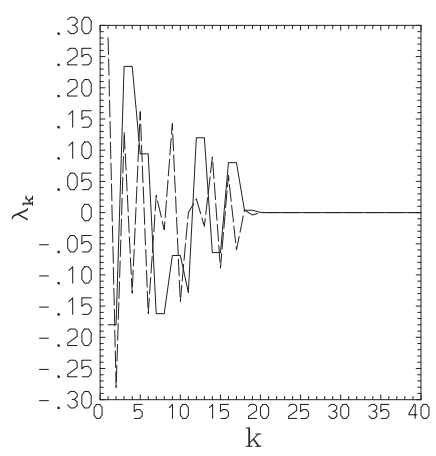

FIG. 9. The eigenvalues $\lambda_{k}$ for the plume matrix $\hat{\boldsymbol{\eta}}_{i j}$, plotted as a function of the index $k$ in decreasing order of their absolute value. Both the real (solid) and imaginary (long dashed) parts are shown. 
(a)

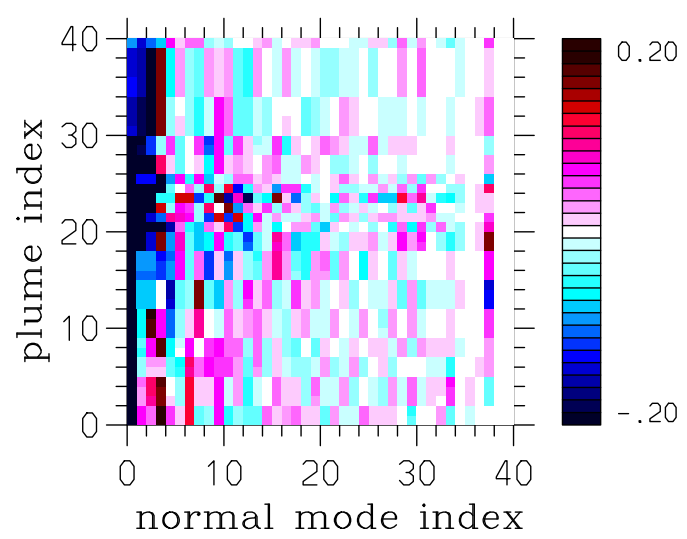

(b)

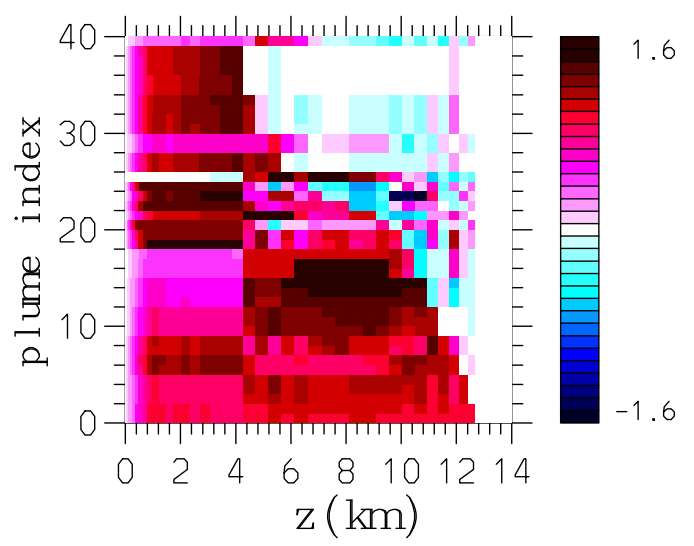

FIG. 10. (a) The plume matrix $\hat{\eta}_{i j}$, as in Fig. $8 \mathrm{~b}$ but here following a regularization by retaining only the first $n_{c}=16$ modes in Eq. (4.4). (b) The vertical profiles for the plume spectrum $\left\{\eta_{j}(z)\right\}$ as in Fig. 8a, but reconstructed after the matrix regularization as in (a). Although both spectra contain complex values, only the real components are shown, the imaginary components being numerically negligible.

original spectrum shown in Fig. 8a; nevertheless, the overall structure remains surprisingly similar.

In summary, the completeness analysis demonstrates the entraining-plume decomposition to be highly redundant, so that it does not directly permit a decomposition of any vertical mass-flux profile under the formulas (4.1) and (4.3) due to a singularity of the matrix $\left(\hat{\eta}_{i j}\right)$. However, the singularity can easily be removed under a singularvector decomposition, and the reconstructed nonsingular plume spectrum remains fairly close to the original entraining-plume spectrum. Thus, the redundancy of the entraining-plume decomposition is not a practical issue in applying the convective quasi-equilibrium closure.

\section{b. Eigenvalues and eigenvectors of the interaction matrix}

The basic structure of the interaction matrix $\mathbf{K}$ can also be elucidated by performing a singular-vector decomposition. Here, the right and the left eigenvectors $\mathbf{M}_{l}$ and $\tilde{\mathbf{M}}_{l}$, respectively, are defined by solving linear eigenvalue problems:

$$
\begin{aligned}
& \mathbf{K M}_{l}=\kappa_{l} \mathbf{M}_{l}, \\
& \tilde{\mathbf{M}}_{l} \mathbf{K}=\kappa_{l} \tilde{\mathbf{M}}_{l},
\end{aligned}
$$

with the eigenvalues $\kappa_{l}(l=1, \ldots, n)$. Recall the orthonormality

$$
\tilde{\mathbf{M}}_{i} \cdot \mathbf{M}_{j}=\delta_{i j} .
$$

The large-scale forcing vector $\mathbf{F}$ may then be represented in terms of the interaction matrix decomposition by

$$
\mathbf{F}=\sum_{l} F_{l} \mathbf{M}_{l}
$$

with the expansion coefficients $F_{l}$ being defined by

$$
F_{l}=\tilde{\mathbf{M}}_{l} \cdot \mathbf{F} .
$$

Similarly, the cloud-base mass-flux vector $\mathbf{M}$ may be represented as

$$
\mathbf{M}=\sum_{l} \mu_{l} \mathbf{M}_{l}
$$

Substitution of Eqs. (4.11) and (4.13) into Eq. (1.1) shows that the expansion coefficients are related by

$$
\mu_{l}=F_{l} / \kappa_{l}
$$

The interaction-matrix eigenvalues $\kappa_{l}$ are plotted in Fig. 11 in decreasing order of their absolute values. From Eq. (4.14), if the large-scale forcing were to contribute with the same order to all of the eigenmodes (cf. Fig. 1b), then the higher-order modes ( $\mathrm{say}, l \geq 14$ ) would dominate the convective response.

Considering the eigenmodes themselves, the spectra of the first eight right and left eigenvectors $\mathbf{M}_{l}$ and $\tilde{\mathbf{M}}_{l}$ are shown in Figs. 12 and 13, respectively. The most striking feature is that the right eigenvectors $\mathbf{M}_{l}$ are dominated by the high-entrainment (i.e., high mode index) shallower modes, whereas the left eigenvectors $\tilde{\mathbf{M}}_{l}$ encompass relatively low-entrainment deep modes (from the eighth to the fourteenth mode index).

These features have significant consequences in defining the response of convection $\mathbf{M}$ against a given large-scale 


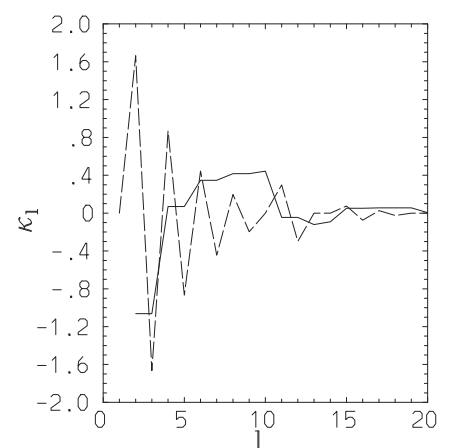

FIG. 11. The eigenvalues $\kappa_{l}$ for the interaction matrix K, plotted as a function of the index $l$ in decreasing order of their absolute value. Both the real (solid) and imaginary (long dashed) parts are shown. An exceptionally large magnitude for the real component of the first eigenvalue (less than -25) is beyond the range of this plot and is not presented. The second to the ninth eigenvalues constitute a series of complex conjugate pairs, as well as the eleventh and the twelfth, and from the fifteenth to the eighteenth eigenvalues.

forcing F. First, the expansion coefficients $F_{l}$ for the large-scale forcing are defined by projecting the large-scale forcing $\mathbf{F}$ onto the left eigenvectors $\tilde{\mathbf{M}}_{l}$ by Eq. (4.12). Since $\tilde{\mathbf{M}}_{l}$ reflects the deeper modes, there is a tendency that the deeper the structure of the large-scale forcing, the stronger the projection onto the expansion coefficients $F_{l}$ and hence onto $\mu_{l}$, through Eq. (4.14). However, the right eigenvectors $\mathbf{M}_{l}$ are dominated by the shallow modes, and thus, the convective response $\mathbf{M}$, as defined by Eq. (4.13) is also dominated by shallow modes. Due to these different characteristics of the left and the right eigenvectors, we therefore find that convection responds most effectively to deeper modes of large-scale forcing, but that it manifests as a response primarily through the shallower modes. Note that this "twisted" relation stems from a strong asymmetry of the interaction matrix, as is demonstrated more explicitly using a simple idealized example matrix in the next subsection.

\section{c. Analysis of an idealized, highly truncated interaction matrix}

It is also informative to take an analytical perspective on the singularities in strict convective quasi-equilibrium closure by examining an idealized interaction matrix, which captures its basic characteristics. Specifically, we consider a $3 \times 3$ interaction matrix $\mathbf{K}$ of the form

$$
\mathbf{K}=\left(\begin{array}{ccc}
k & -k_{d} & -k_{d} \\
k_{s} & k & -k_{d} \\
k_{s} & k_{s} & -1
\end{array}\right) .
$$
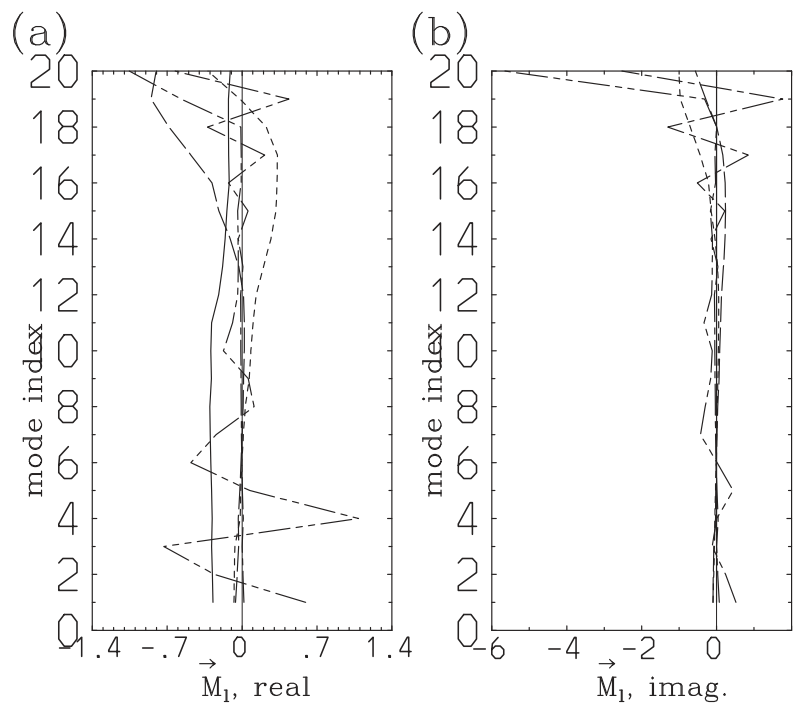

FIG. 12. The first eight right eigenvectors $\mathbf{M}_{l}(l=1, \ldots, 8)$ of the interaction matrix, as defined by Eq. (4.9a). (a) Real and (b) imaginary components. The first four vectors are shown by solid, long-dashed, short-dashed, and dot-dashed curves. They are followed by four other varying types of the curves. Note the change of scale in the horizontal axis.

The quasi-equilibrium closure of Eq. (1.1) reduces to

$$
\mathbf{K}\left(\begin{array}{l}
M_{3} \\
M_{2} \\
M_{1}
\end{array}\right)+\left(\begin{array}{l}
F_{3} \\
F_{2} \\
F_{1}
\end{array}\right)=0 .
$$

Here, the order of the vector indices for $\mathbf{M}$ and $\mathbf{K}$ is reversed from a standard convention so that the matrix form defined by Eq. (4.15a) closely follows the matrixelement distributions shown in Fig. 4: the given distribution can directly be compared with the definition Eq. (4.15a) by flipping the horizontal direction in the figures. The idealized matrix is normalized by setting the right-lowest element to $-1 ; k, k_{s}$, and $k_{d}$ are expected to be small values, where $k$ and $k_{s}$ represent destabilization tendencies of shallow convection modes acting on themselves and on the deeper modes, respectively, whereas $-k_{d}$ represents the stabilization from the deeper modes to shallower modes.

The solution to the matrix problem (4.15b) is

$$
\begin{aligned}
M_{3}= & {\left[\left(2 k-k_{s}+k_{d}-1\right) k_{s} k_{d}-k^{2}\right]^{-1}\left[\left(k-k_{s} k_{d}\right) F_{3}\right.} \\
& \left.+\left(1+k_{s}\right) k_{d} F_{2}-\left(k+k_{d}\right) k_{d} F_{1}\right],
\end{aligned}
$$

$$
\begin{aligned}
M_{2}= & {\left[\left(2 k-k_{s}+k_{d}-1\right) k_{s} k_{d}-k^{2}\right]^{-1}\left[\left(k_{d}-1\right) k_{s} F_{3}\right.} \\
& \left.+\left(k-k_{s} k_{d}\right) F_{2}-\left(k-k_{s}\right) k_{d} F_{1}\right],
\end{aligned}
$$



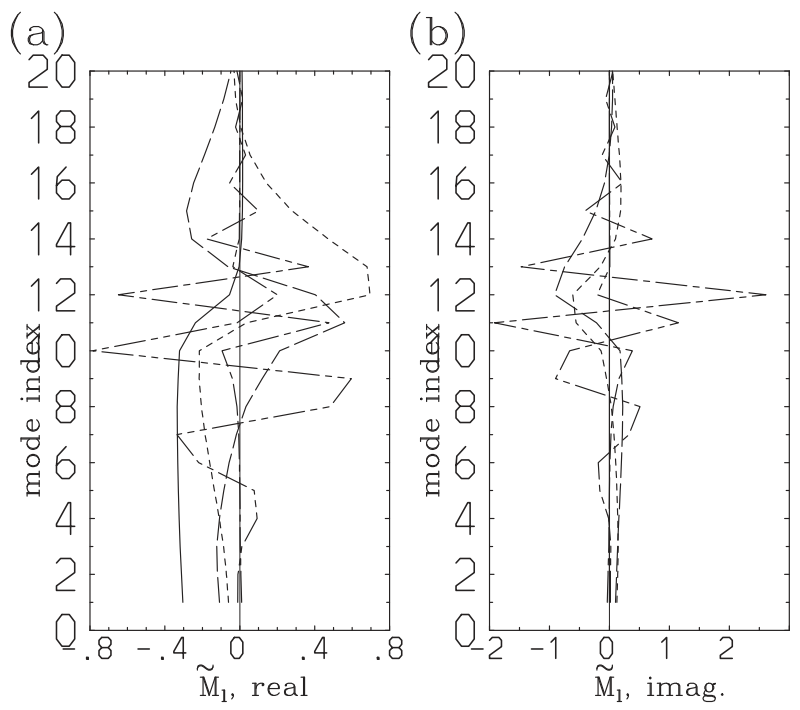

FIG. 13. The first eight left eigenvectors $\tilde{\mathbf{M}}_{l}(l=1, \ldots, 8)$ of the interaction matrix, as defined by Eq. (4.9b). Plotted in the same format as for the right eigenvectors in Fig. 12.

$$
\begin{aligned}
M_{1}= & {\left[\left(2 k-k_{s}+k_{d}-1\right) k_{s} k_{d}-k^{2}\right]^{-1}\left[\left(k-k_{s}\right) k_{s} F_{3}\right.} \\
& \left.+\left(k+k_{d}\right) k_{s} F_{2}-\left(k^{2}+k_{s} k_{d}\right) F_{1}\right] .
\end{aligned}
$$

A further simplification is to set all the small parameters to the same value, $k_{s}=k_{d}=k$, so that the solution in Eq. (4.16) becomes

$$
\begin{aligned}
& M_{3}=\frac{1}{2(k-1) k}\left[(1-k) F_{3}+(1+k) F_{2}-2 k F_{1}\right], \\
& M_{2}=\frac{F_{3}-F_{2}}{2 k} \\
& M_{1}=\frac{-F_{2}+F_{1}}{1-k} .
\end{aligned}
$$

When the limit of $k \rightarrow 0$ is taken, the above solution reduces to

$$
\begin{aligned}
& M_{3} \rightarrow-\frac{F_{3}+F_{2}}{2 k}, \\
& M_{2} \rightarrow \frac{F_{3}-F_{2}}{2 k}, \\
& M_{1} \rightarrow-F_{2}+F_{1},
\end{aligned}
$$

retaining only the leading terms with respect to $k$, and assuming all forcing components $F_{j}(j=1,2,3)$ to be of $O(1)$. Thus, the two shallowest convective modes, $M_{3}$ and $M_{2}$, respectively, diverge in the limit of $k \rightarrow 0$. Also note that the signs of $M_{2}$ and $M_{1}$ sensitively depend on differences between $F_{3}$ and $F_{2}$, and that between $F_{2}$ and $F_{1}$, respectively.

In this manner, the idealized matrix (4.15a) provides a very simple demonstration for the origin of the singular behaviors of the quasi-equilibrium closure that were seen in previous subsections.

\section{d. Perturbation analysis}

The idealized matrix problem may be further developed by considering a perturbation expansion. Noting that many of the matrix elements are small in $\mathbf{K}$, we can write

$$
\begin{aligned}
\mathbf{K} & =\mathbf{K}^{(0)}+\delta \mathbf{K}^{(1)}, \\
\mathbf{M} & =\mathbf{M}^{(0)}+\delta \mathbf{M}^{(1)}+\ldots,
\end{aligned}
$$

where $\delta$ is a small expansion parameter, and where the idealized matrix (4.15a) can be decomposed as

$$
\begin{aligned}
\mathbf{K}^{(0)} & =\left(\begin{array}{lll}
0 & 0 & 0 \\
0 & 0 & 0 \\
0 & 0 & 1
\end{array}\right), \\
\delta \mathbf{K}^{(1)} & =\left(\begin{array}{ccc}
k & -k_{d} & -k_{d} \\
k_{s} & k & -k_{d} \\
k_{s} & k_{s} & 0
\end{array}\right) .
\end{aligned}
$$

To $O(1)$, we obtain

$$
\mathbf{K}^{(0)} \mathbf{M}^{(0)}+\mathbf{F}=0,
$$

with $\operatorname{det}\left[\mathbf{K}^{(0)}\right]=0$, because of the fact that large elements are localized, and hence there is no solution available for $\mathbf{M}^{(0)}$. To avoid this problem, we need to reformulate the expansion of Eq. (4.18b) as

$$
\mathbf{M}=\frac{1}{\delta} \mathbf{M}^{(-1)}+\mathbf{M}^{(0)}+\ldots
$$

so that we obtain to $O(1 / \delta)$ :

$$
\mathbf{K}^{(0)} \mathbf{M}^{(-1)}=0,
$$

which, with the matrix (4.19a), leads to

$$
M_{1}^{(-1)}=0
$$

and leaves the other two components $M_{2}^{(-1)}$ and $M_{3}^{(-1)}$ as undetermined. At $O(1)$ we have

$$
\mathbf{K}^{(0)} \mathbf{M}^{(0)}+\mathbf{K}^{(1)} \mathbf{M}^{(-1)}+\mathbf{F}=0,
$$

which makes the problem solvable. Specifically for the case with Eqs. (4.19a) and (4.19b), this $O(1)$ relation defines $M_{2}^{(-1)}, M_{3}^{(-1)}$, and $M_{1}^{(0)}$. 
Thus, the perturbation analysis here more explicitly demonstrates how a strict application of the convective quasi-equilibrium condition tends to lead to an abnormally strong response of shallow convection to largescale forcing.

\section{Physical implications}

The present paper has focused on a rather narrow question of mathematical difficulties with the original closure formulation of Arakawa and Schubert (1974). In concluding, we turn to the physical implications from the present findings, also referring to background issues.

\section{a. Free-ride principle}

The convective quasi-equilibrium closure of Eq. (1.1) is based on stationarity of the cloud work function, which is a vertically integrated quantity [cf. Eq. (133) of Arakawa and Schubert 1974]. Thus, the closure is also formulated in terms of vertically integrated quantities. However, we might intuitively expect that a certain quasi-equilibrium state (i.e., a balance condition) is achieved at each vertical level, at least to a good approximation, if a large enough number of convective modes is considered. The different modes provide different weighting functions and upper limits for the integrals in question.

It is observationally known that the large-scale tropical atmosphere satisfies a free-ride state [Fraedrich and McBride 1989; later Sobel et al. (2001) term it alternatively as "weak temperature gradient"], with a close balance between the large-scale tendency and the convective response in both the heat and moisture equations:

$$
\begin{aligned}
w \frac{d \bar{\theta}}{d z} & \simeq Q_{1}+Q_{R}, \\
w \frac{d \bar{q}_{v}}{d z} & \simeq-\frac{C_{p}}{L} Q_{2} .
\end{aligned}
$$

Here, $Q_{1}$ and $Q_{2}$ are tendencies due to nonadvective processes, apart from radiative heating, $Q_{R}$, in the context of large-scale modeling (i.e., convective-scale advections are not explicitly considered). See Fig. 1 of Yano (2001; also reproduced as Fig. 4.2 in chapter 4 of Plant and Yano 2015) for a graphical demonstration. Although the literature tends to refer only to the balance (5.1a), here, it is seen that the second balance Eq. (5.1b) is equally valid. In the large-scale tropical atmosphere, $Q_{1}$ and $Q_{2}$ are mostly due to convection (i.e., $Q_{c}$ ). On the other hand, the vertical advection and the radiation terms may be combined to define the total large-scale forcing $F_{L}$. Thus, the free-ride state may be equivalently expressed in the form

$$
Q_{c}+F_{L} \simeq 0
$$

for both variables. Equation (5.2) may be considered as a statement of convective quasi equilibrium, but defined separately on each vertical level, rather than as an integral constraint.

Hence, we are led to ask whether, given enough plume modes in Eq. (1.1), we obtain a free-ride state corresponding to Eq. (5.2): Will this be actually accomplished in practice by the quasi-equilibrium closure?

\section{b. Completeness of the plume spectrum}

Equivalence between Eqs. (1.1) and (5.2) could be established if the mass-flux spectrum were able to represent any possible convective response that may be required to satisfy the free-ride state. Thus, a first consideration is whether the mass-flux spectrum is flexible enough to represent any possible vertical profile. This has been examined using normal-mode and singularvector decompositions in section $4 \mathrm{a}$. The entrainingplume decomposition is shown to be highly redundant, as expected from the individual plume profiles (cf. Fig. 2), and so a decomposition of the entraining plumes into normal modes does not provide well-defined expansion coefficients. However, this ill posedness of the decomposition can be resolved by removing all the singular vectors with almost-vanishing eigenvalues from the expansion. A reconstructed plume spectrum still remains fairly close to the original entraining-plume spectrum, but practically removing the redundancy.

Here, the mathematical question of the completeness of a plume spectrum addresses its capacity and flexibility to represent any physically feasible vertical structure of convection. As we have seen, the conclusion obtained is rather mixed, and further investigations from a more practical perspective could be warranted.

\section{c. Convective response under the spectrum mass flux}

The next consideration is how an individual plume mode modifies the large-scale thermodynamic state (i.e., convective response: section $3 \mathrm{~b}$ ). The effect of an individual entraining plume is composed of two main parts: (i) detrainment that cools and moistens the large scale by reevaporation of the detrained cloudy air and (ii) compensating environmental descent, in response to the convective updraft, that induces adiabatic heating and drying. A major difference between these two effects is that the detrainment effect is found only at a single level at the plume top, whereas the environmental descent is felt at all of the vertical levels spanned by the plume. 
As a result, the detrainment effect focused on a single vertical level tends to be abnormally strong, with cooling and moistening rates far exceeding $10 \mathrm{Kday}^{-1}$ and so strongly dominant at that level over the environmentaldescent effect.

The consequence is that a straightforward inversion of the interaction matrix in the closure condition of Eq. (1.1) produces a full convective response against a given large-scale forcing that becomes very singular (section 3c). For idealized large-scale forcing profiles with a half-sine-shaped large-scale uplifting, we find that the convective response is dominated by singularly strong warming and cooling induced at the top of the detraining-plume downdrafts (i.e., entraining-plume updraft modes with a negative amplitude). Due to the tendency of entraining-plume modes to produce a singular response, the convective quasi-equilibrium closure condition does not achieve a thermodynamic state close to the free-ride balances. Thus, the mathematical analysis herein points out in an explicit manner how and why a physically unrealistic feature of the entrainingplume model causes a problem.

A very simple way of removing these singular cooling-moistening effects would be to neglect all of the detrainment effects from the interaction matrix $\mathbf{K}$ by setting $\mathbf{K}_{d}=0$ in Eq. (3.1) so that the interaction matrix $\mathbf{K}$ is replaced by $\mathbf{K}_{v}$. However, totally removing this effect from the convective equilibrium problem would not be very realistic for reasons discussed in section $4 \mathrm{e}$.

\section{d. Interaction matrix analysis}

Another important aspect of the convective response under the convective quasi-equilibrium closure is the dominance of shallow plumes regardless of the vertical extent of large-scale forcing. This is rather unintuitive. However, one must remember that as a matter of principle, large-scale forcing is projected to all the plume modes by design, as explicitly shown by Fig. 1a. The resulting spectrum of the convective response is rather nontrivial, mathematically taking the form of a matrix inversion. This character of the problem means that we need to pay attention to the mathematical behavior of the inversion calculation in order to better understand the structural issues involved.

First, a singular-vector decomposition is performed on the interaction matrix in section $4 \mathrm{~b}$. The left-eigenvector spectra are dominated by middle-height plume modes, with maximum heights of $8-10 \mathrm{~km}$; thus, relatively deep components of large-scale forcing lead to a strong response by convection. On the other hand, the righteigenvector spectra are dominated by shallow plume modes, and thus, relatively deep large-scale forcing modes are strongly projected onto shallow convective modes.

This rather strong asymmetry between the left and the right eigenvectors stems from a strong asymmetry in the interaction matrix itself. In turn, the asymmetry of the interaction matrix stems from the nature of the detrainment effect of a plume mode onto other plume modes: only the deeper plume modes are affected by detrainment from a given plume mode, and this gives rise to the triangular structure apparent within the interaction matrix (cf. Fig. 4b).

With increasing precipitating efficiency, the detrainment effect becomes weaker as less cloudy air is available to detrain at plume top. In a fully precipitating limit for all of the plume modes, then the asymmetry of the interaction matrix would disappear, and the singular response to the large-scale forcing would be removed. However, additional calculations (not shown) indicate that even a weak asymmetry of the interaction matrix can lead to a singular response. A relatively strong sensitivity of the convective response to the transition scale $\varepsilon_{0}$ in precipitation efficiency [Eq. (2.5)] has also been found because this parameter controls the relative contribution of detrainment effects to the interaction matrix.

An idealized $3 \times 3$ interaction matrix (sections $4 \mathrm{c}$ and 4d) is able to reproduce the character of these results. A singular perturbation expansion is required for describing the convective quasi-equilibrium closure due the fact that the matrix elements related to shallow convection tend to be substantially smaller than those for the interactions between deep convection. As a result, shallow convection tends to respond to large-scale forcing in a singular manner.

\section{e. Further physical implications}

An important feature throughout the present analysis is the strong cooling and moistening induced by reevaporation of the detrained cloudy air. When this contribution is suppressed, the convective response under the quasi-equilibrium closure becomes much more reasonable. It is worth noting that some alternative formulations of mixing, beyond the simple entrainment formulation of pure Arakawa and Schubert (1974), may help to alleviate the problem (de Rooy et al. 2013; Yano 2015). Another legitimate way of suppressing this effect is to couple the convection parameterization with a stratiform cloud representation, and to transfer the detrained cloudy convective air to form part of a stratiform cloud rather than immediately reevaporating it into the environment. The importance of this procedure would probably be needless to emphasize, because such a coupling of convection with stratiform clouds is 
accomplished in most of the operational global models already. However, its significance, to the extent revealed here, appears to be not widely appreciated.

At the same time, completely suppressing the evaporative cooling of the detrained cloudy air would likely not be wise. Yano and Plant (2012b) suggest that the resulting destabilization tendency of shallow convection can be a key mechanism driving transformations from shallow to deep convection. Two solutions may be considered for this remedy. The first is to retain the tendency explicitly for shallow convection, rather than imposing a strict equilibrium constraint. In this case, a singular response of shallow convection to large-scale forcing associated with evaporative cooling must be tamed in a different manner. The second is to transfer the role of this destabilization tendency to the stratiform cloud scheme: the mechanism may be represented by the cloud-top entrainment process (cf. Deardorff 1980; Randall 1980) under this reformulation, which is also expected to lead to an equivalent destabilization.

Another important implication from the present study is a much slower response time scale for the shallower convective modes than for the deep convection, as indicated by the relatively small elements in the interaction matrix. This implication can be seen directly from the prognostic equation for the cloud work function spectrum A from which the quasi-equilibrium closure (1.1) is derived:

$$
\frac{\partial \mathbf{A}}{\partial t}=\mathbf{K M}+\mathbf{F} .
$$

The quasi-equilibrium closure has been justified based on an argument that an overall time scale for the response of convection to large-scale forcing is so short that we can drop the time tendency of the cloud work function on the left-hand side, which is expected to evolve by following a slow large-scale time scale.

However, more precisely, the response time scale is short only for deep convection, but not for shallow convection. As a result, Eq. (5.3) may be approximated by Eq. (1.1) for the deep convection part only. In other words, the full convective ensemble does not immediately respond to any slow large-scale forcing, as originally envisioned by Arakawa and Schubert (1974). Rather, a finite time scale for the convective response to large-scale forcing should explicitly be taken into account by retaining the temporal tendency of the cloud work function on the left-hand side of Eq. (5.3), so that the closure becomes fully prognostic. Suitable formulations are already in place (e.g., Pan and Randall 1998; Yano and Plant 2012c). Here, we point out a solid reason for moving toward this direction.
The issues appear to be further involved, because observational analyses by Zhang (2002, 2003), Donner and Phillips (2003) suggest that the boundary layer processes controlling the evolution of the convective available potential energy (CAPE), and thus also likely of the cloud work functions, are of a much shorter time scale than those found in the free atmosphere. Thus, boundary layer processes, neglected in the analysis herein, may further contribute to break down a strict application of convective quasi-equilibrium closure. Those implications warrant further investigations.

The present study further suggests needs for reconsidering the mass-flux convection parameterization formulation from more general perspectives. Such investigations are already under way (e.g., Yano et al. 2005b; Yano 2014b, 2016). These developments should more seriously be considered in operational contexts.

\section{APPENDIX}

\section{Scale Analysis}

The purpose of this appendix is to estimate the order of magnitude of cooling and moistening associated with reevaporation of the detrained cloudy air.

We begin with the simple point that latent heating due to the condensation of a unit of water vapor, $q=1 \mathrm{~g} \mathrm{~kg}^{-1}$, leads to an increase of temperature by

$$
q L / C_{p}=2.5 \mathrm{~K}
$$

using the latent heating, $L=2.5 \times 10^{6} \mathrm{~J} \mathrm{~kg}^{-1}$, and the specific heat capacity, $C_{p}=10^{3} \mathrm{~J} \mathrm{~kg}^{-1} \mathrm{~K}^{-1}$ for air at constant pressure. A typical mass-flux value under convective quasi equilibrium is $M \sim 10^{-2} \mathrm{~kg} \mathrm{~m}^{-2} \mathrm{~s}^{-1}$, or $M / \rho \sim w_{0}=10^{-2} \mathrm{~m} \mathrm{~s}^{-1}$ in units of vertical velocity. At the convective cloud top, all of the mass flux detrains under the entraining-plume hypothesis. The associated heat flux is thus

$$
\begin{aligned}
(M / \rho)\left(q_{c} L / C_{p}\right) & \sim 2.5 \times 10^{-2} \times q_{c} \mathrm{~K} \mathrm{~m} \mathrm{~s}^{-1} \\
& \sim 2.5 \times 10^{3} \times q_{c} \mathrm{~K} \mathrm{~m} \mathrm{day}^{-1},
\end{aligned}
$$

where the detrained cloud water mixing ratio $q_{c}$ is expressed in units of $\mathrm{g} \mathrm{kg}^{-1}$. If the detrainment occurs over a layer of, say, $1 \mathrm{~km}$ in depth, it will amount to a cooling rate of $2.5 \mathrm{~K} \mathrm{day}^{-1}$ for $q_{c}=1 \mathrm{~g} \mathrm{~kg}^{-1}$.

The last piece of estimate is the amount of cloud water, $q_{c}$, expected at the convective cloud top at the height of, say, $H \sim 10 \mathrm{~km}$. To obtain this, we note that within a convective updraft, condensative heating is well balanced by adiabatic cooling [a local realization of freeride state: cf. Eq. (5.1)]. Thus, 


$$
\frac{L}{C_{p}} \frac{d q_{v}^{*}}{d z}+\frac{d \theta}{d z} \simeq 0
$$

This relation leads to an estimate for the rate of decrease of saturated water vapor with height,

$$
\frac{d q^{*}}{d z} \sim-\frac{C_{p}}{L} \frac{d \theta}{d z} \sim-10^{-6} \mathrm{~m}^{-1}
$$

Thus, neglecting fall out due to precipitation, the accumulation of condensed water in lifting through a height $H \sim 10 \mathrm{~km}$ is estimated as

$$
\begin{aligned}
q^{*}= & -H \frac{d q_{V}^{*}}{d z} \sim 10^{-6} \mathrm{~m}^{-1} \times 10^{4} \mathrm{~m} \\
& \sim 10^{-2} \mathrm{~kg} \mathrm{~kg}^{-1} \sim 10 \mathrm{~g} \mathrm{~kg}^{-1}
\end{aligned}
$$

Substitution of Eq. (A.2) into Eq. (A.1) leads to an estimate of the cooling rate of $25 \mathrm{~K} \mathrm{day}^{-1}$ for a $1-\mathrm{km}$-deep detrainment layer.

\section{REFERENCES}

Arakawa, A., and W. H. Schubert, 1974: Interaction of a cumulus cloud ensemble with the large-scale environment, part I. J. Atmos. Sci., 31, 674-701, https://doi.org/10.1175/15200469(1974)031<0674:IOACCE > 2.0.CO;2.

Bechtold, P., N. Semane, P. Lopez, J.-P. Chaboureau, A. Beljaars, and N. Bormann, 2014: Representing equilibrium and nonequilibrium convection in large-scale models. J. Atmos. Sci., 71, 734-753, https://doi.org/10.1175/JAS-D-13-0163.1.

Deardorff, J. W., 1980: Cloud top entrainment instability. J. Atmos. Sci., 37, 131-147, https://doi.org/10.1175/1520-0469(1980) 037<0131:CTEI >2.0.CO;2.

de Rooy, W. C., and Coauthors, 2013: Entrainment and detrainment in cumulus convection: An overview. Quart. J. Roy. Meteor. Soc., 139, 1-19, https://doi.org/10.1002/qj.1959.

Donner, L. J., and V. T. Phillips, 2003: Boundary layer control on convective available potential energy: Implications for cumulus parameterization. J. Geophys. Res., 108, 4701, https:// doi.org/10.1029/2003JD003773.

Fraedrich, K., and J. L. McBride, 1989: The physical mechanism of CISK and the free-ride balance. J. Atmos. Sci., 46, 2642-2648, https://doi.org/10.1175/1520-0469(1989)046<2642:TPMOCA > 2.0.CO;2.

Fulton, S. R., and W. H. Schubert, 1985: Vertical normal mode transforms: Theory and application. Mon. Wea. Rev., 113, 647-658, https://doi.org/10.1175/1520-0493(1985) 113<0647:VNMTTA $>2.0$.CO;2.

Hack, J. J., W. H. Schubert, and P. L. Silva Dias, 1984: A spectral cumulus parameterization for use in numerical models of tropical atmosphere. Mon. Wea. Rev., 112, 704-716, https://doi.org/ 10.1175/1520-0493(1984)112<0704:ASCPFU>2.0.CO;2.

Houze, R. A., Jr., and A. K. Betts, 1981: Convection in GATE. Rev. Geophys. Space Phys., 19, 541-576, https://doi.org/10.1029/ RG019i004p00541.

Jordan, C. L., 1958: Mean sounding for the West Indies area. J. Meteor., 15, 91-97, https://doi.org/10.1175/1520-0469(1958) 015<0091:MSFTWI $>2.0 . \mathrm{CO} ; 2$.
Kasahara, A., and K. Puri, 1981: Spectral representation of threedimensional global data by expansion in normal mode functions. Mon. Wea. Rev., 109, 37-51, https://doi.org/10.1175/ 1520-0493(1981)109<0037:SROTDG > 2.0.CO;2.

Lord, S. J., 1982: Interaction of a cumulus cloud ensemble with the large-scale environment. Part III: Semi-prognostic test of the Arakawa-Schubert cumulus parameterization. J. Atmos. Sci., 39, 88-103, https://doi.org/10.1175/1520-0469(1982)039<0088: IOACCE $>2.0 . \mathrm{CO} ; 2$.

— semble with the large-scale environment. Part II. J. Atmos. Sci., 37, 2677-2692, https://doi.org/10.1175/1520-0469(1980) 037<2677:IOACCE $>2.0 . \mathrm{CO} ; 2$.

—_, W. C. Chao, and A. Arakawa, 1982: Interaction of a cumulus cloud ensemble with the large-scale environment. Part IV: The discrete model. J. Atmos. Sci., 39, 104-113, https://doi.org/10.1175/1520-0469(1982)039<0104:IOACCE > 2.0.CO;2.

Mapes, B. E., 1997: Equilibrium vs. activation controls on largescale variations of tropical deep convection. The Physics and Parameterization of Moist Atmospheric Convection, R. K. Smith, Ed., Kluwer Academic, 321-358.

Moorthi, S., and M. J. Suarez, 1992: Relaxed Arakawa-Schubert: A parameterization of moist convection for general circulation models. Mon. Wea. Rev., 120, 978-1002, https://doi.org/10.1175/ 1520-0493(1992)120<0978:RASAPO > 2.0.CO;2.

Pan, D.-M., and D. A. Randall, 1998: A cumulus parameterization with prognostic closure. Quart. J. Roy. Meteor. Soc., 124, 949-981, https://doi.org/10.1002/QJ.49712454714.

Plant, R. S., and J.-I. Yano, Eds., 2015: Parameterization of Atmospheric Convection, World Scientific, 1172 pp., http:// www.worldscientific.com/worldscibooks/10.1142/p1005.

Randall, D. A., 1980: Conditional instability of the first kind upside down. J. Atmos. Sci., 37, 125-130, https://doi.org/ 10.1175/1520-0469(1980)037<0125:CIOTFK >2.0.CO;2.

Sobel, A. H., J. Nilsson, and L. M. Polvani, 2001: The weak temperature gradient approximation and balanced tropical moisture waves. J. Atmos. Sci., 58, 3650-3665, https://doi.org/ 10.1175/1520-0469(2001)058<3650:TWTGAA > 2.0.CO;2.

Yano, J.-I., 2001: Residual cumulus parameterization. Quart. J. Roy. Meteor. Soc., 127, 1261-1276, https://doi.org/10.1002/ qj.49712757407.

_ 2014a: Basic convective element: Bubble or plume? A historical review. Atmos. Phys. Chem., 14, 7019-7030, https:// doi.org/10.5194/acp-14-7019-2014.

_ 2014b: Formulation structure of mass-flux convection parameterization. Dyn. Atmos. Oceans, 67, 1-28, https://doi.org/ 10.1016/j.dynatmoce.2014.04.002.

_ 2015: Thermodynamic effects of convection under the massflux formulation. Theoretical Background and Formulation, R. S. Plant and J. I. Yano, Eds., Vol. 1, Parameterization of Atmospheric Convection, World Scientific, 227-248.

_ 2016: Subgrid-scale physical parameterization in atmospheric modelling: How can we make it consistent? J. Phys., 49A, 284001, https://doi.org/10.1088/1751-8113/49/28/284001.

_ , and R. S. Plant, 2012a: Convective quasi-equilibrium. Rev. Geophys., 50, RG4004, https://doi.org/10.1029/2011RG000378. , and - 2012b: Interactions between shallow and deep convection under a finite departure from convective quasi equilibrium. J. Atmos. Sci., 69, 3463-3470, https://doi.org/ 10.1175/JAS-D-12-0108.1.

, and — 2012c: Finite departure from convective quasiequilibrium: Periodic cycle and discharge-recharge mechanism. 
Quart. J. Roy. Meteor. Soc., 138, 626-637, https://doi.org/10.1002/ qj. 957.

- , and —, 2016: Generalized convective quasi-equilibrium principle. Dyn. Atmos. Oceans, 73, 10-33, https://doi.org/ 10.1016/j.dynatmoce.2015.11.001.

_, J.-P. Chaboureau, and F. Guichard, 2005a: A generalization of CAPE into potential-energy convertibility. Quart. J. Roy. Meteor. Soc., 131, 861-875, https://doi.org/10.1256/qj.03.188.

, J.-L. Redelsperger, F. Guichard, and P. Bechtold, 2005b: Mode decomposition as a methodology for developing convectivescale representations in global models. Quart. J. Roy. Meteor. Soc., 131, 2313-2336, https://doi.org/10.1256/qj.04.44.
— - M. Bister, Z. Fuchs, L. Gerard, V. Phillips, S. Barkidija, and J. M. Piriou, 2013: Phenomenology of convectionparameterization closure. Atmos. Phys. Chem., 13, 4111-4131, https://doi.org/10.5194/acp-13-4111-2013.

Zhang, G. J., 2002: Convective quasi-equilibrium in midlatitude continental environment and its effect on convective parameterization. J. Geophys. Res., 107, 4220, https://doi.org/ 10.1029/2001JD001005.

2003: The concept of convective quasi-equilibrium in the tropical western Pacific: Comparison with midlatitude continental environment. J. Geophys. Res., 108, 4592, https:// doi.org/10.1029/2003JD003520. 